\title{
Scheduling Single-Machine Problem Oriented by Just-In-Time Principles - A Case Study
}

\author{
${ }^{1}$ Joana D. Dantas, ${ }^{2}$ Leonilde R. Varela \\ Department of Production and Systems \\ School of Engineering \\ University of Minho \\ Azurém Campus Guimarães, Portugal \\ 1joanadantas91@gmail.com, ${ }^{2}$ leonilde@dps.uminho.pt
}

\author{
Ana Maria Madureira \\ GECAD Research Group \\ School of Engineering \\ Polytechnic Institute of Porto \\ Porto, Portugal \\ amd@isep.ipp.pt
}

\begin{abstract}
Developments in advanced autonomous production resources have increased the interest in the Single-Machine Scheduling Problem (SMSP). Until now, researchers used SMSP with little to no practical application in industry, but with the introduction of multi-purpose machines, able of executing an entire task, such as 3D Printers, replacing extensive production chains, single-machine problems are becoming a central point of interest in real-world scheduling. In this paper we study how simple, easy to implement, Just-in-Time (JIT) based, constructive heuristics, can be used to optimize customer and enterprise oriented performance measures. Customer oriented performance measures are mainly related to the accomplishment of due dates while enterprise-oriented ones typically consider other timeoriented measures.
\end{abstract}

Keywords - Single-Machine Scheduling Problem; Scheduling; Perfomance Measures; Just-in-Time; Comparative Analysis; Heuristics.

\section{INTRODUCTION}

Manufacturing scheduling consists of an important production management function, affecting all branches of industrial, commercial and services organizations. In the strict industrial context, a very important objective, which is usually imposed by clients, consists on satisfying due dates for manufacturing orders.

With the developments in Computer Numerical Control (CNC) machines and the advanced computer assisted manufacturing techniques, situations on real-world environments involving single-machine scheduling problems (SMSP) have increased. SMSP can also be viewed as base problems, usually treated in the context of more complex manufacturing environments, for instance in job shops, for solving bottleneck problems [1-4]. Therefore, single-machine scheduling problems are also important while trying to solve more complex ones, as the solution for the whole complex scheduling problem can be found through a decomposition [3, 5-7]. Many studies have been carried out over decades about SMSP [8-23]. In these problems not only the assumptions and specificities can be different, but also the objective that is intended to be analysed, which can led to quite difficult problems to solve [8, 11-16, 22, 23].

Moreover, SMSP also remain quite promising and interesting scheduling problems to be explored due to its great potential for dealing with several distinct performance measures. Performance measures can be generally classified as external or costumer-oriented (measures related to the client, such as lead times or due dates) [8-11] or internal or enterpriseoriented, such as makespan, total completion time, and earliness among others [12-16]. Both types of performance measures are important to be considered, and can be treated jointly, under the principles of the Just-in-Time (JIT) philosophy [19-23]. The Just-in-Time philosophy tries to minimize the earliness and the lateness at the same time. In the beginning most of scheduling research were focused on singlecriteria problems, as can be seen in [17] and [18]. However, as organizations are concerned with the request from multiple stakeholders, it is necessary that clients and enterprise are both satisfied. Although special attention should be given to the costumer-oriented performance measures, internal interests also have to be taken into account.

In this paper two different approaches for SMSP, based on external and internal performance measures, as described and applied to a problem presented and treated in the literature as well as a comparative analysis is performed. In order to clearly refer to the main subjects underlying this work, the paper is organized as follows. Section II defines the scheduling problem treated in this work. Section III briefly describes several different scheduling resolution approaches, including the algorithms used in this research. Section IV presents the results obtained and a comparative analysis with results from the same problem presented in literature. Finally, section V presents some conclusions and future work.

\section{SCHEDULING PROBLEM}

The scheduling problem considered in this study consists of a SMSP with $n$ jobs to be processed in order to follow, as close as possible, a JIT strategy. This strategy intends to minimize the number of tardy jobs, while also being concerned with minimizing the sum of costs across all jobs (earliness and tardiness), among other more secondary performance measures, which are also very important in scheduling approaches, in general, and in JIT-based approaches in particular.

The problem of minimizing the sum of tardiness and earliness reflects the interest of minimizing inventory holding costs and maximizing client approval trough due date 
satisfaction. Additional performance measures can relate to the maximum completion time of jobs or makespan $\left(\mathrm{C}_{\max }\right)$ and the total completion time of jobs $\left(\sum \mathrm{C}_{\mathrm{i}}\right)$, which although being internal or enterprise-oriented performance measures, as stated before, are also very important.

Therefore, we still have to pay attention to them too, as a balanced approach, which intends to reach solutions that try to obtain balanced schedules in terms of external and internal objectives is of upmost importance. Special attention should be given to this balance, which is even more important due to the actual economic crisis that we are still experiencing.

\section{A. Nomenclature and assumptions}

The SMSP studied will follow some assumptions:

- $\quad$ All the jobs are available at time zero;

- The machine can process at most one job at a time;

- $\quad$ No preemptions are allowed;

- Associated to each job $j(j=1,2, \ldots n)$ there is a processing time $p_{j}$ and a due date $d_{j}$.

Therefore, the problem can be classified as $1\left|p_{j}, d_{j}\right| N t, T_{\max }$, $C_{\text {max }}$, and $E_{\text {max }}$, and also $\left(\sum T_{i}\right.$ and $T_{\text {mean }}, \sum C_{i}$ and $C_{\text {mean }}, \sum E_{i}$ and $\left.E_{\text {mean }}\right)$, according to $\alpha|\beta| \gamma$ nomenclature [24].

\section{B. Dominance properties/objectives}

In this paper, we will present a comparative analysis based on several different performance measures referred bellow, which are either associated to external or internal objectives:

- $\mathrm{N}_{\mathrm{t}}$ - number of tardy jobs

- $\mathrm{T}_{\max }-$ maximum tardiness

- $\mathrm{C}_{\max }-$ maximum completion time

- $\mathrm{E}_{\max }-$ maximum earliness

- $\quad \sum \mathrm{T}_{\mathrm{j}}, \mathrm{T}_{\text {mean }}-$ total and mean tardiness

- $\quad \sum \mathrm{C}_{\mathrm{j}}, \mathrm{C}_{\text {mean }}$ - total and mean completion time

- $\quad \sum \mathrm{E}_{\mathrm{j}}, \mathrm{E}_{\text {mean }}-$ total and mean earliness

We intend to analyse all this different performance measures and try to reach a balance, to find a good, or at least a satisfactory solution for the analysed problem, under the scope of the proposed JIT principles.

\section{SCHEDULING OPTIMIZATION APPROACHES AND ALGORITHMS}

Most scheduling problems can be solved optimally, provided that enough computational time is available. Often, they can be formulated as a (mixed) integer programming problem. Due to the NP-completeness nature that these class of problems, complete enumeration of all possibilities quickly becomes prohibitively time consuming $[25,26]$. However, in some situations, efficient branch and bound methods, dynamic programming techniques, and integer programming may provide optimal solutions for reasonable problem sizes in reasonable time [27]. In [16] authors surveys several of these and other more complex scheduling approaches and systems, namely for real-time, distributed and dynamic scheduling, which can also be even more complex scenarios when some process for dealing with uncertainty is incorporated [2-5, 7, 2830].

If the computational time required for obtaining optimal solutions is beyond reasonable, it is necessary to use heuristic solutions, like dispatching procedures (which usually do not yield optimal solutions). Dispatching procedures with priority rules is one of the best known optimization technique for scheduling, and is surveyed in detail in [27]. Using dispatching rules schedules can be produced almost instantaneously. Because of their simplicity, they are also used quite often in industry and in flexible manufacturing and assembly systems. Dispatching rules, however tend to yield a low and unpredictable performance. For some simple scheduling problems, efficient algorithms do exist to calculate the optimal solutions [1, 8, 13, 23, 31].

For instance, the earliest due date dispatching rule (EDD) provides an optimal solution for the minimisation of the maximum tardiness for the single-machine scheduling problem. However, this is not the only one, the shortest processing time dispatching rule (SPT), in a single-machine problem, lead to a minimal mean completion time and mean lateness. This rule sequenced the jobs in a processing time increasing order [32].

Usually, real-life problems are more complex than these academic problems, but sometimes, by using a simplified model and applying the results to the real situation, the scheduling problem can be successfully solved (e.g., scheduling a factory with a single bottleneck as a single processor scheduling problem $[1,2,5]$.

AI-based approaches for scheduling, sometimes known as knowledge-based scheduling approaches, use specific knowledge about the scheduling problem in their decision process [3, 4, 6, 7, 33]. For instance, rule-based approaches store scheduling rules (entered by humans) into a knowledge based and some web-based systems for supporting manufacturing scheduling decision making, which enable to solve quite different scheduling problems, varying from single-machine environments up to more complex ones, like job shops, manufacturing cells or other kind of flexible manufacturing systems [34-37].

In the context of AI-based approaches for manufacturing scheduling, meta-heuristics are one of the most important branches; meta-heuristics such as simulated annealing, tabu search and genetic algorithms have been deeply studied [1, 5, 29, 38]. Neighbourhood search techniques (NST) are a simplification of meta-heuristics which are also used for MS, as they are quite easy to implement and to use, while enabling good solutions to be reached, especially when dealing with some more simple scheduling problems, like single stage or single-machine problems $[17,39,40]$.

Usually near-optimal approaches enable to obtain a higher performance than simple dispatching rules, but they also use a considerable amount of computational time [17]. Therefore, the selection of a scheduling algorithm depends on a trade-off between computational time and schedule performance.

This paper used a software tool to collect the computational results. The software used includes two different approaches, a Hodgson's algorithm adaptation (HAA) and a Neighbourhood Search Technique (NST), which are going to be better described in the next sub-section.

\section{A. Hodgson algorithm adaptation (HAA)}

The Hodgson algorithm (HA) determines the sequence of tasks in which the number of tardy jobs is minimal [16, 20, 22, 23]. Let $E$ be the set that contains all the jobs that must be processed and $L$ the set of all late jobs, which starts empty. Thus, the algorithm integrates the following sequence of steps: 
Step 1: Sort the jobs that belong to the $E$ set and sort them by increasing delivery date (earliest due date rule, EDD).

Step 2: If none of the jobs is delayed the sequence is optimal. If the opposite happens, the delayed job at its $k$ position (i.e. [k]) is identified.

Step 3: Identify the entity or job with the bigger duration or processing time $\left(\mathrm{p}_{\mathrm{j}}\right)$ in the set of the first $k$ jobs. Remove it from the set $E$ and put it into the $L$ set. Establish the new time for the conclusion of the remaining jobs on $E$ and return to step 2 .

There are several priority rules, which can be used for establishing an initial solution as a starting point for the Hodgson's algorithm, which will act as a rule that specifies the priority on how the jobs present in the waiting queue of a machine are processed. The Earliest Due Date (EDD) rule specifies that when a machine is free, the job that has the earliest due date to be processed first is selected.

This work used a Hodgson's algorithm adaptation (HAA) implementation, by using JAVA 5 language [34, 41]. As described before, the Hodgson's algorithm (and HAA) ensures the sequence with the least number of delays or tardy jobs in a single-machine environment [17].

\section{B. Neighbourhood Search Techniques (NST)}

The Neighbourhood Search Techniques (NST) is a local search technique included in the scope of heuristic approaches that perform a neighbourhood search for the local optimal solution (or local optimum); and they usually are fast, simple to implement and flexible $[17,34,41]$. Associated to this kind of methods it turns out to be necessary to specify a mechanism, to create an initial solution or seed, which consists upon a neighbourhood generation mechanism, and also a criterion for selecting the next seed and a stopping criterion $[17,34,41]$.

For some problems, efficient NST-based algorithms exist $[17,34,41]$, but for most, optimal algorithms, require excessive calculation time [17]. If the optimal solution cannot be found in reasonable time, heuristics or meta-heuristics should be used [17, 34, 39-41]. The simplest heuristics are based on simple dispatching rules [17, 34, 39-41]. Several other heuristics are built on artificial intelligence (AI) techniques $[1,3,4,6,7,17,28,29,33-35,39-42]$. Neighbourhood search techniques, and particularly metaheuristics, have also provided some powerful algorithms for near-optimal schedules [17, 39, 40].

The NST procedure used in this work includes the following steps [34, 41]:

Step 1: Select a seed solution for evaluating its performance.

Step 2: Generate and evaluate solutions in the neighbourhood of the seed. If none of the solutions produces better performance than the seed, then the search ends. Otherwise continue.

Step 3: Select the solution from the neighbourhood with best performance for being the new seed.

Moreover, it is necessary to specify the following procedures [34, 41]:

1. A method for obtaining a seed.
2. A certain mechanism for generating the neighbourhood.

3. A method for selecting the solution that will be the next seed.

The HAA and the NST algorithms implementation used in this work enable to obtain two different sequences of jobs and resulted in quite good solutions, which are going to be described further in the next section. They are also compared to the SPT dispatching rule.

\section{COMPUTATIONAL STUDY}

The work presented in this paper is a continuation of a previous work [43] with an instance of five jobs for the problem presented in section III. In this paper we intend to generalize that work. To generalize the problem analysis, we apply the same two methods, NST and HAA and the dispatching rule SPT, to fifty-six dimensions increasing the number of jobs, being from five to ninety nine.

The evolution of problems results is a linear growth, for most of the performance measures. The results obtained for Hodgson Algorithm application appear to be the more effective for some of the performance measures, like minimizing the tardiness and the earliness, along with the total and mean earliness. However the mean earliness is only better for large problems with more than twenty jobs.

The NST and SPT application to these problems reached better results for the total and mean tardiness and completion time and the mean earliness did perform better for small problems with less than twenty jobs.

For all methods, the maximum completion time is the same, being the minimum for the respective size of problem. This is because none of them does introduce idle times between jobs for a minimized earliness, producing a sequence of jobs without any forced interruption.

\section{A. Irregular Performance Measures Analysis}

To obtain a better analysis of the considered problem, we did observe three irregular performance measures:

- Difference between the maximum tardiness and maximum earliness;

- Difference between the mean completion time and its optimal value

- The sum of the two previous performance measures.

In the first perfomance measure, which includes the maximum tardiness and the maximum lateness, we can observe that the methods performances are similar and the difference between the maximum tardiness and the maximum lateness is directly proportional to the number of jobs in the problem.

In the second performance measure, the Hodgson solutions obtained a similar behavior as the previous performance measure but the SPT performance was null, since this dispatching rule guarantees an optimal mean completion time, and there is no difference between the mean completion time found and its optimal value. The NST also obtained a null or a very low value regarding this performance measure, which means that the NST obtained an optimal value for the mean completion time or very close to the optimum. 
In the last performance measures all three optimization techniques obtained similar results.

\section{B. Correlation with the number of jobs in the problem}

We analysed, through the use of SPSS, the correlation between these three performance measures and the number of jobs in the problem, by using the Pearson Correlation method.

In the next table we can analyze the correlation results obtained through the SPSS software. We can conclude with $95 \%$ of confidence that there is a correlation, except for the Cmean-Cmean* results through the use of the SPT and the NST algorithms. Regarding the case of the SPT results it is impossible to realize the correlation since the performance measure evaluated is a constant (zero). For the case of the NST results the correlation did not exist, as the results are near-optimal for all problem sizes.

TABLE I. RESULTS FOR CORRELATION ANALYSIS

\begin{tabular}{|c|c|c|c|}
\hline $\begin{array}{c}\text { Performance } \\
\text { measures }\end{array}$ & SPT & HAA & NST \\
\hline Tmax-Emax & 0.997 & 0.998 & 0.997 \\
\hline Cmean-Cmean* & - & 0.978 & -0.238 \\
\hline Formula & 0.997 & 0.998 & 0.997 \\
\hline
\end{tabular}

\section{Performance Evaluation}

The performance of each method was evaluated through the ANOVA methodology (Table II).

TABLE II. RESUSTS OF ANOVA

\begin{tabular}{|c|c|}
\hline Performance Measure & Sig. \\
\hline Tmax-Emax & 0.890 \\
\hline Cmean-Cmean* & 0.000 \\
\hline Formula & 0.662 \\
\hline
\end{tabular}

The performance obtained is similar, with the same exception already referred about the mean completion time performance measure $\left(\mathrm{C}_{\text {mean }}-\mathrm{C}_{\text {mean }}{ }^{*}\right)$. For the $\mathrm{T}_{\max }-\mathrm{E}_{\mathrm{ma}} \mathrm{x}$, the ANOVA does indicate that there is a variance equality, which confirms an existence of similar results for the three methods (SPT, HAA and NST). The other performance measure (Table II), continues to verify the exception case, which seems natural, considering the performance of the SPT and the NST. So the ANOVA does indicate that we reject the hypothesis of the variances equality of the methods results. For the formula that encloses the previous two performance measures there is variance equality, so we can accept the hypothesis about a similar methods performance.

\section{Evaluation of the methods performance with size differenciation}

To differentiate the behavior between the small problems and the big ones, we considered the following categorization:

\section{TABLE III. DIFFERENT DIMENSIONS OF PROBLEMS}

\begin{tabular}{|c|c|}
\hline Dimension & Size of problems \\
\hline Small & $\leq 20$ \\
\hline Big & $>20$ \\
\hline
\end{tabular}

As there are no more than thirty problems for the problems with a small dimension to be able to assume its normal distribution, it is necessary to perform a test that proves it. So we performed a Shapiro-Wilk test, and obtained the results presented on Table IV. As we can realize, it is not possible to reject our null hypothesis with a 95\% confidence level, data following a normal distribution.

\section{TABLE IV. RESULTS FOR SHAPIRO-WILK TEST}

\begin{tabular}{|c|c|}
\hline Algorithm & Sig. \\
\hline SPT & 0.856 \\
\hline HAA & 0.534 \\
\hline NST & 0.609 \\
\hline
\end{tabular}

For one performance measure one of the methods reveals a better performance than the other. With the same evaluation of the previous point but taking into consideration the size differentiation for all three performance measures, we have been analyzing, we can conclude that the methods performances are similar.

The obtained results are systematized on Tables V and VI for smaller and bigger problems.

We can only reject with $95 \%$ confidence that all methods performances, for the Cmean-Cmean*, are not identical. In the other performance we assume that all three methods achieve similar results.

TABLE V. RESULTS OF ANOVA FOR SMALL PROBLEMS

\begin{tabular}{|l|c|}
\hline \multicolumn{1}{|c|}{ Performance Measure } & Sig. \\
\hline Tmax-Emax & 0.942 \\
\hline Cmean-Cmean* & 0.000 \\
\hline Formula & 0.813 \\
\hline
\end{tabular}

TABLE VI. RESULTS OF ANOVA FOR BIG PROBLEMS

\begin{tabular}{|l|c|}
\hline \multicolumn{1}{|c|}{ Performance Measure } & Sig. \\
\hline Tmax-Emax & 0.766 \\
\hline Cmean-Cmean* & 0.000 \\
\hline Formula & 0.393 \\
\hline
\end{tabular}

Most times, the difference that turns out a method more effective than another, for one performance measure is not significant, regarding that the performance differences between methods are statistically insignificant. Therefore, we have to analyze more than one performance measure, like in $[16,21-23]$ and by using more than one approach like in [2023]. However, we can also enclose three performance measures, each one with different perspectives, in one single equation, allowing a more global and faster analysis.

Moreover, we also analyze the performance measures behavior with the size growth of the problem, like in $[8,14$, $16,21]$ and this permit to conclude about what to expect about the methods performance for problems with different sizes. 


\section{CONCLUSIONS}

In this paper an implementation of the Hodgson's method and a NST algorithm were proposed for solving single-machine scheduling problem and a comparative analysis was performed. The results obtained were compared to the ones referred in literature and our results proved to be more effective, while enabling to reach a more balanced solution in terms of customer and enterprise oriented performance measures, by means of JIT principles, although even more importance was given to customer-oriented measures, namely to the total number of tardy jobs and the maximum tardiness.

The obtained results permits to conclude that most times minimizing the earliness may deteriorate the tardiness results. Therefore, it is of upmost importance to keep attention to all the important performance measures, for instance, external or customer-oriented ones, and realize that we cannot give the same importance to performance measures such as the earliness and the tardiness, as they may yield different results.

Through the generalization of the problem, despite the increase of the value of almost all performance measures, their growth was proportional to the growth of number of jobs in the problem. The only exception was the performance of the NST for the mean completion time, which was optimal or very close to the optimal. The methods performances are similar regardless of the problem size.

In future work, we intend to apply other methods in order to compare them with the obtained results, for the performance measures considered, and even extend the analysis for other important performance measures, also for larger problems, with an increase in the number of jobs and further extending the statistical tests for obtaining an improved generalized analysis about the problems' performance measures behavior.

\section{ACKNOWLEDGMENT}

The authors wish to acknowledge the support of the Fundação para a Ciência e Tecnologia (FCT), Portugal, through the grant "Projeto Estratégico - UI 252 - 2011-2012" reference PEst-OE/EME/UI0252/2011 and FCOMP-01-0124FEDER-PEst-OE/EEI/UI0760/2014.

\section{REFERENCES}

[1] T. Pinto and L. R. Varela, "Comparing Extended Neighborhood Seardh Techniques Applied to Production Scheduling"," The Romanian Review Precision Mechanics, Optics \& Mechatronics, vol. 20, 2010.

[2] A. Madureira, C. Ramos, and S. C. Silva, "Resourceoriented scheduling for real world manufacturing systems," in IEEE International Symposium, 2003, pp. 140-145.

[3] A. Madureira, N. Sousa, and I. Pereira, "Selforganization for Scheduling in Agile Manufacturing," presented at the 10th IEEE International Conference on Cybernautic Intelligent Systems 2011, London, United Kingdom, 2011.

[4] A. Madureira, I. Pereira, P. Pereira, and A. Abraham, "Negotiation mechanism for self- organized scheduling system with collective intelligence," Neurocomputing, vol. 132, pp. 97-110, 2014.
[5] A. Madureira, C. Ramos, and S. D. do Carmo Silva, "A coordination mechanism for real world scheduling problems using genetic algorithms," vol. 1, ed, 2002, pp. $175-180$.

[6] A. Madureira and J. Santos, "Proposal of multi-agent based model for dynamic sheduling in manufacturing," WSEAS Transacions on Information Science \& Applications, vol. 2, 2005.

[7] A. Madureira and I. Pereira, "Intelligent Bio- Inspired system for manufacturing scheduling under uncertainties," ed, 2010, pp. 109-112.

[8] H. Hamidreza, A. Mohammad Ebrahim, and P. Keyvan Kamandani, "A branch and bound for single machine stochastic scheduling to minimize the maximum lateness," International Journal of Industrial Engineering Computations, vol. 3, p. 499, 2012.

[9] L. Liu and H. Zhou, "Applying Variable Neighborhood Search to the Single- machine Maximum Lateness Rescheduling Problem," Electronic Notes in Discrete Mathematics, vol. 39, pp. 107-114, 2012.

[10] A. Subramanian, M. Battarra, and C. N. Potts, "An Iterated Local Search heuristic for the single machine total weighted tardiness scheduling problem with sequence-dependent setup times," International Journal of Production Research, vol. 52, pp. 27292742, 2014.

[11] M. Mohammad Mahdavi, H. Hamidreza, M. Mohammad, and A. Kazem, "A calibrated hybrid for the single machine scheduling problem with objective of minimizing total weighted tardiness with dependent setup," International Journal of Information, Business and Management, vol. 4, p. 55, 2012.

[12] G. Mosheiov and U. Yovel, "Minimizing weighted earliness- tardiness and due-date cost with unit processing-time jobs," European Journal of Operational Research, vol. 172, pp. 528-544, 2006.

[13] C. C. Lu, S. W. Lin, K. C. Ying, C.-C. Lu, S.-W. Lin, and K.-C. Ying, "Robust scheduling on a single machine to minimize total flow time.(Report)," Computers \&amp; Operations Research, vol. 39, p. 1682, 2012.

[14] L. Jiyin and B. L. MacCarthy, "Effective heuristics for the single machine sequencing problem with ready times," International Journal of Production Research, vol. 29, p. 1521, 1991.

[15] C. Y. Low, M. Ji, C. J. Hsu, and C. T. Su, "Minimizing the makespan in a single machine scheduling problems with flexible and periodic maintenance," Appl. Math. Model., vol. 34, pp. 334-342, 2010.

[16] U. Al-Turki, C. Fedjki, and A. Andijani, "Tabu search for a class of single- machine scheduling problems," Computers and Operations Research, vol. 28, pp. 1223-1230, 2001.

[17] M. Pinedo, Scheduling: theory, algorithms, and systems: Prentice Hall, 2002.

[18] P. Brucker, Scheduling Algorithms: Springer, 2007.

[19] S. Pathumnakul and P. J. Egbelu, "Algorithm for minimizing weighted earliness penalty in singlemachine problem," European Journal of Operational Research, vol. 161, p. 780, 2005.

[20] W. Y. Chen and G. J. Sheen, "Single- machine scheduling with multiple performance measures: 
Minimizing job-dependent earliness and tardiness subject to the number of tardy jobs," International Journal of Production Economics, vol. 109, pp. 214229, 2007.

[21] W. S. Yoo and L. A. Martin-Vega, "Scheduling singlemachine problems for on- time delivery," Comput. Ind. Eng., vol. 39, pp. 371-392, 2001.

[22] Y. Huo, J. Y. T. Leung, and H. Zhao, "Bi- criteria scheduling problems: Number of tardy jobs and maximum weighted tardiness," European Journal of Operational Research, vol. 177, pp. 116-134, 2007.

[23] G. Wan and B. P. C. Yen, "Single machine scheduling to minimize total weighted earliness subject to minimal number of tardy jobs," European Journal of Operational Research, vol. 195, pp. 89-97, 2009.

[24] M. L. R. Varela and S. do Carmo Silva, "An ontology for a model of manufacturing scheduling problems to be solved on the web," in Innovation in Manufacturing Networks, ed: Springer, 2008, pp. 197-204.

[25] R. V. Rao and P. Vivek, "An elitist teaching-learningbased optimization algorithm for solving complex constrained optimization problems," International Journal of Industrial Engineering Computations, vol. 3, p. 535, 2012.

[26] M. Babaei, M. Mohammadi, S. M. T. F. Ghomi, and M. A. Sobhanallahi, "Two parameter-tuned metaheuristic algorithms for the multi-level lot sizing and scheduling problem," International Journal of Industrial Engineering Computations, vol. 3, p. 751, 2012.

[27] D. Ouelhadj and S. Petrovic, "A survey of dynamic scheduling in manufacturing systems," J Sched, vol. 12, pp. 417-431, 2009.

[28] A. Madureira, "Hybrid Meta-heuristics based system for Distributed Dynamic Scheduling," in Encyclopedia of Artificial Intelligence, J. R. Rabuñal, J. Dorado, and A. Prazos, Eds., Idea Group Reference, Information Science Reference ed.

[29] L. R. Varela and R. A. Ribeiro, "Evaluation of simulated annealing to solve fuzzy optimization problems," Journal of Intelligent and Fuzzy Systems, vol. 14, pp. 59-71, 2003.

[30] M. L. R. Varela, Ribeiro, R. A., "Distributed Manufacturing Scheduling based on a Dynamic MultiCriteria Decision Model," in 2nd World Conference on Soft Computing, 2012.

[31] Z. Liu, "Single machine scheduling to minimize maximum lateness subject to release dates and precedence constraints," Computers and Operations Research, vol. 37, pp. 1537-1543, 2010.

[32] J. Jungwattanakit, M. Reodecha, P. Chaovalitwongse, and F. Werner, "Algorithms for flexible flow shop problems with unrelated parallel machines, setup times, and dual criteria," Int. J. Adv. Manuf. Technol., vol. 37, pp. 354-370, 2008.

[33] A. Madureira, J. Santos, and I. Pereira, "Hybrid Intelligent System For Distributed Dynamic Scheduling," Springer-Verlag, Ed., ed, 2009.

[34] M. L. R. Varela, G. D. Putnik, and M. M. Cruz-Cunha, "Web-based Technologies Integration for Distributed Manufacturing Scheduling in a Virtual Enterprise,"
International Journal of Web Portals (IJWP), vol. 4, pp. 19-34, 2012.

[35] M. L. R. Varela, R. Barbosa, and G. Putnik, "Experimental Platform for Collaborative Inter and Intra Cellular Fuzzy Scheduling in an Ubiquitous Manufacturing System," Communications in Computer and Information Science series, pp. 227-236, 2012.

[36] J. B. Carvalho, M. L. R. Varela, G. D. Putnik, J. E. Hernándes, and R. A. Ribeiro, "Title," unpublished|.

[37] A. Arrais-Castro, M. L. R. Varela, J. J. Jassbi, R. A. Ribeiro, and F. C. C. Dargam, "Title," unpublished|.

[38] T. Ghosh, S. Sengupta, M. Chattopadhyay, and P. K. Dan, "Meta-heuristics in cellular manufacturing: A state-of-the-art review," International Journal of Industrial Engineering Computations, vol. 2, pp. 87 122, 2011.

[39] A. Nareyek, Local Search for Planning and Scheduling: ECAI 2000 Workshop, Berlin, Germany, August 21, 2000. Revised Papers: Springer, 2001.

[40] F. Xhafa and A. Abraham, Metaheuristics for Scheduling in Industrial and Manufacturing Applications: Springer, 2008.

[41] G. G. Vieira, M. L. R. Varela, and G. Putnik, "Technologies Integration for Distributed Manufacturing Scheduling in a Virtual Enterprise," Communications in Computer and Information Science series, pp. 345-355, 2012.

[42] R. A. Ribeiro and L. R. Varela, "Fuzzy optimization using simulated annealing: An example set," in Fuzzy Sets Based Heuristics for Optimization, ed: Springer, 2003, pp. 159-180.

[43] J. D. Dantas and L. R. Varela, "Scheduling singlemachine problem based on just-in-time principles," presented at the Nature and Biologically Inspired Computing, 2014. 